\title{
Dynamics of evaporative cooling in magnetically trapped atomic hydrogen
}

\author{
Makoto Yamashita, ${ }^{1}$ Masato Koashi, ${ }^{2}$ Tetsuya Mukai, ${ }^{1}$ Masaharu Mitsunaga, ${ }^{1, *}$ and Nobuyuki Imoto ${ }^{1,2}$ \\ ${ }^{1}$ NTT Basic Research Laboratories, 3-1, Morinosato Wakamiya, \\ Atsugi-shi, Kanagawa 243-0198, Japan \\ ${ }^{2}$ The Graduate University for Advanced Studies, Shonan Village, \\ Hayama, Kanagawa 240-0193, Japan
}

(November 15, 2018)

We study the evaporative cooling of magnetically trapped atomic hydrogen on the basis of the kinetic theory of a Bose gas. The dynamics of trapped atoms is described by the coupled differential equations, considering both the evaporation and dipolar spin relaxation processes. The numerical time-evolution calculations quantitatively agree with the recent experiment of Bose-Einstein condensation with atomic hydrogen. It is demonstrated that the balance between evaporative cooling and heating due to dipolar relaxation limits the number of condensates to $9 \times 10^{8}$ and the corresponding condensate fraction to a small value of $4 \%$ as observed experimentally.

Pacs. 03.75.Fi, 05.20.Dd, 32.80.Pj

The recent development of atom-manipulation techniques [i] has realized Bose-Einstein condensation (BEC) in magnetically trapped alkali-metal atoms $[2,1$ and atomic hydrogen [5]. In all these experiments, evaporative cooling was adopted at the final stage of the cooling procedures and was essential in obtaining the extremely low temperatures needed for the quantum degeneracy. The cooling mechanism of this powerful method is based on both the selective removal of energetic atoms through evaporation and collisional rethermalizations among the remaining atoms [6]. Evaporative cooling itself is stimulating theoretical studies of the process of condensate formation in nonequilibrium atomic gases 17 17.

In BEC experiments with atomic hydrogen, evaporative cooling has been implemented by lowering the potential height of the saddle point at one end of a magnetic trap with cylindrical symmetry [1]. This approach suffers from a reduction of the dimension of evaporation at low temperatures due to low elastic collision rate 18,14 since the $s$-wave scattering length, $a$, of a hydrogen atom is anomalously small, about two orders of magnitude smaller than that in alkali-metal atoms. Heating caused by the dipolar spin relaxation then easily retards the evaporative cooling and prevents further cooling before reaching the critical temperature of BEC. To over-

\footnotetext{
*Present address: Department of Physics, Faculty of Science, Kumamoto University, Kurokami 2-39-1, Kumamoto 860-8555, Japan.
}

come this problem, Fried et al. adopted the evaporative cooling induced by radio-frequency (rf) magnetic field just after the "saddle-point" evaporative cooling [5]. The atom ejection technique utilizing the transition between trapped and untrapped spin states enables efficient threedimensional evaporation even at low temperatures and in a highly anisotropic magnetic potential [6]. BEC was finally achieved as a result of the heating-cooling balance in this efficient "rf-induced" evaporative cooling.

There are several characteristic features of BEC in atomic hydrogen as compared with that in alkali-metal atoms [5]. The small mass, $m$, of hydrogen resulted in the transition temperature of $50 \mu \mathrm{K}$, the highest among BEC experiments. While the condensate fraction, i.e., the ratio of condensates to the whole trapped atoms, remained a small value of about $5 \%$, a huge number of condensates containing about $10^{9}$ atoms were observed and the peak density of the condensates was about 25 times higher than that of the noncondensed atoms. Furthermore, with respect to the loss of condensates, both the three-body recombination process and the background gas collisions in a cryogenic environment of trap are negligible in the hydrogen system. Only dipolar spin relaxation becomes the dominant loss mechanism, and causes serious heating [19,20].

In this paper, the kinetic theory for evaporative cooling of a magnetically trapped Bose gas [15] is extended to include dipolar relaxation loss. This theory enables the consistent and quantitative investigations on the whole evaporative cooling process of the experiments, from the classical regime at the early stage of cooling to the quantum degenerate regime after the BEC transition. We applied it to the rf-induced evaporative cooling in the recent BEC experiment with atomic hydrogen [0] and quantitatively investigated the characteristic features of hydrogen BEC mentioned above. We expect further that our calculation studying the dynamics of trapped atoms in cooling process will give a good estimation for the growing of condensates in the experiment, since there is room for discussions on the number of condensates measured in the spectroscopic way $[5]$.

Here, we briefly mention the formulation of the theory 15. During evaporative cooling, the applied rf-magnetic field effectively truncates the trapping potential, $U(\mathbf{r})$, at the energy, $\epsilon_{t}$, determined by the rf-field frequency. The thermalized distribution of noncondensed atoms in such a truncated potential is well approximated by the 
truncated Bose-Einstein distribution function

$$
\tilde{f}(\mathbf{r}, p)=\frac{1}{\tilde{\xi}^{-1}(\mathbf{r}) \cdot \exp \left(\epsilon_{p} / k_{B} T\right)-1} \cdot \Theta\left(A(\mathbf{r})-\epsilon_{p}\right),
$$

where $T$ is the temperature, $\epsilon_{p}=p^{2} / 2 m$ is the kinetic energy of atoms, and $\Theta(x)$ is the step function. $\tilde{\xi}(\mathbf{r})$ denotes the local fugacity including the mean field potential energy; $\tilde{\xi}(\mathbf{r})=\exp \left\{[\mu-U(\mathbf{r})-2 v \tilde{n}(\mathbf{r})] / k_{B} T\right\}$, where $\mu$ is the chemical potential, $v=4 \pi a \hbar^{2} / m$ is interaction strength between trapped atoms, and $\tilde{n}(\mathbf{r})$ is the density profile of atoms in a truncated potential. The step function in Eq. (II) eliminates the momentum states whose kinetic energies exceed the effective potential height $A(\mathbf{r})=\epsilon_{t}-U(\mathbf{r})-2 v \tilde{n}(\mathbf{r})$. The momentum integration of this truncated distribution function gives the density profile in a self-consistent way such that $\tilde{n}(\mathbf{r})=4 \pi h^{-3} \int \tilde{f}(\mathbf{r}, p) p^{2} \mathrm{~d} p$, and similarly the internal energy density such that $\tilde{e}(\mathbf{r})=4 \pi h^{-3} \int \epsilon_{p} \tilde{f}(\mathbf{r}, p) p^{2} \mathrm{~d} p+$ $v \tilde{n}^{2}(\mathbf{r})+U(\mathbf{r}) \tilde{n}(\mathbf{r})$. The total number of atoms, $\tilde{N}$, and the total internal energy, $\tilde{E}$, in a truncated potential are evaluated respectively by the spatial integrations of these density functions, $\tilde{N}=\int \tilde{n}(\mathbf{r}) \mathrm{d} \mathbf{r}$ and $\tilde{E}=\int \tilde{e}(\mathbf{r}) \mathrm{d} \mathbf{r}$. At low temperatures, the density profile in the condensed region is described as the sum of condensates, $n_{0}(\mathbf{r})$, and saturated noncondensed atoms, $\tilde{n}_{n}$, such that $\tilde{n}(\mathbf{r})=n_{0}(\mathbf{r})+\tilde{n}_{n}$. Condensates obey the Thomas-Fermi distribution $n_{0}(\mathbf{r})=n_{p}-U(\mathbf{r}) / v$, where $n_{p}$ represents the peak density of condensates at the center of the potential. The density $\tilde{n}_{n}$ is evaluated through the truncated Bose-Einstein distribution function under the condition $\tilde{\xi}(\mathbf{r})=1$.

The dynamics of trapped atoms during evaporative cooling is investigated on the basis of kinetic theory of a Bose gas [15]. Hydrogen atoms are removed from the trapping potential through both evaporation and dipolar spin relaxation processes [5, 19,20]. The change rates (i.e., loss rates) of density functions, $\dot{n}_{\text {loss }}$ and $\dot{e}_{\text {loss }}$, are evaluated respectively as the sum of the contributions of both processes, such that

$$
\begin{aligned}
& \dot{n}_{\mathrm{loss}}(\mathbf{r})=-\dot{n}_{\mathrm{ev}}(\mathbf{r})-G_{2} \cdot K(\mathbf{r}) \cdot \tilde{n}^{2}(\mathbf{r}), \\
& \dot{e}_{\mathrm{loss}}(\mathbf{r})=-\dot{e}_{\mathrm{ev}}(\mathbf{r})-G_{2} \cdot K(\mathbf{r}) \cdot \tilde{e}(\mathbf{r}) \cdot \tilde{n}(\mathbf{r}),
\end{aligned}
$$

where $\dot{n}_{\mathrm{ev}}$ and $\dot{e}_{\mathrm{ev}}$ are the evaporation rates of density functions, $G_{2}$ is the dipolar decay rate constant, and $K$ is the correlation function which describes the secondorder coherence of trapped atoms 2022]. Both $\dot{n}_{\mathrm{ev}}$ and $\dot{e}_{\text {ev }}$ were derived from a general collision integral of a Bose gas system in Ref. [15], and we adopt here the opposite sign of the notations of these rates defined in this reference. The bosonic feature of evaporation process is therefore included as the strong enhancement of these evaporation rates due to the stimulated scattering of atoms. Regarding the dipolar spin relaxation process, we assume the correlation function of an ideal Bose gas such that $K(\mathbf{r})=\left(n_{0}^{2}(\mathbf{r})+4 n_{0}(\mathbf{r}) \tilde{n}_{n}+2 \tilde{n}_{n}^{2}\right) / 2 \tilde{n}^{2}(\mathbf{r})$ [20 22]. The spatial integration of Eq. (2) gives important thermodynamic quantities such as the loss rate of total number of trapped atoms, $\dot{N}_{\text {loss }}$, and that of the total internal energy, $\dot{E}_{\text {loss }}$ :

$$
\begin{aligned}
& \dot{N}_{\text {loss }}=\int \dot{n}_{\text {loss }}(\mathbf{r}) \mathrm{d} \mathbf{r}=-\dot{N}_{\mathrm{ev}}-\dot{N}_{\mathrm{dip}}, \\
& \dot{E}_{\mathrm{loss}}=\int \dot{e}_{\mathrm{loss}}(\mathbf{r}) \mathrm{d} \mathbf{r}=-\dot{E}_{\mathrm{ev}}-\dot{E}_{\mathrm{dip}},
\end{aligned}
$$

where $\dot{N}_{\text {ev }}\left(\dot{E}_{\text {ev }}\right)$ and $\dot{N}_{\text {dip }}\left(\dot{E}_{\text {dip }}\right)$ represent the evaporation rate and dipolar relaxation loss rate, respectively. These loss rates are complicated functions of temperature $T$, chemical potential $\mu$, and truncation energy $\epsilon_{t}$. Finally, the system obeys the coupled differential equations

$$
\frac{\partial \tilde{N}}{\partial t}=\dot{N}_{\text {loss }}, \quad \frac{\partial \tilde{E}}{\partial t}=\dot{E}_{\text {loss }}
$$

Time-evolution calculations were performed numerically on the assumption of quick rethermalization, which is appropriate for the slow evaporative cooling used in the usual BEC experiments [15]. Thus, the system always stays in a quasi-thermal equilibrium state and is described by the truncated Bose-Einstein distribution function in Eq. (1).

Precise knowledge of experimental conditions is also necessary for our quantitative calculations. The magnetic trap adopted in the BEC experiment with atomic hydrogen [5] is modeled by the Ioffe-Pritchard potential $U(\rho, z)=\sqrt{(\alpha \rho)^{2}+\left(\beta z^{2}+\theta\right)^{2}}-\theta$ with the radial potential gradient $\alpha=2.2 \times 10^{-23} \mathrm{~J} / \mathrm{m}$, the axial curvature $2 \beta=6.8 \times 10^{-24} \mathrm{~J} / \mathrm{m}^{2}$, and the bias energy $\theta=$ $4.8 \times 10^{-28} \mathrm{~J}$ (i.e., $\theta / k_{\mathrm{B}}=35 \mu \mathrm{K}$ ). At low energies, this potential is well approximated by the harmonic one with the radial oscillation frequencies $\omega_{\rho}=\alpha / \sqrt{m \theta}=2 \pi \times 3.9$ $\mathrm{kHz}$ and the axial frequency $\omega_{z}=\sqrt{2 \beta / m}=2 \pi \times 10 \mathrm{~Hz}$. It should be noted here that, even after the BEC transition, many noncondensed hydrogen atoms still distribute in the high-energy region, where such harmonic approximation fails. We treated the Ioffe-Pritchard potential exactly for the quantitative description of noncondensed atoms. Accordingly, all density functions were calculated in the cylindrical coordinate.

As is well known, the efficiency of evaporative cooling strongly depends on the way the rf-field frequency is swept (i.e., forced evaporative cooling) [6]. The high efficiency is obtained by the slow evaporative cooling which continues for the time on the order of 10 seconds. In the BEC experiment with atomic hydrogen, the rf-field frequency was swept according to the functional form 23] $\nu(t)=\nu_{\mathrm{i}}\left(\frac{\nu_{\mathrm{f}}}{\nu_{\mathrm{i}}}\right)^{\tau^{g}}$, where $\nu_{\mathrm{i}}=23 \mathrm{MHz}$ is the initial rffrequency, $\nu_{\mathrm{f}}=2 \mathrm{MHz}$ is the final one, $\tau=t / t_{0}$ denotes the normalized time with the specific time $t_{0}=25 \mathrm{~s}$, and $g=1.5$ is the fixed parameter. The truncation energy $\epsilon_{t}$ then changes according to the relation $\epsilon_{t}(t)=h \nu(t)-\theta$. 
Furthermore, we employed the following experimental parameters [5]: $s$-wave scattering length $a=6.48 \times 10^{-2}$ $\mathrm{nm}$ [24], the dipolar decay rate constant $G_{2}=1.1 \times 10^{-15}$ $\mathrm{cm}^{3} / \mathrm{s}$ [19], initial temperature $T=120 \mu \mathrm{K}$, initial peak density $\tilde{n}(\mathbf{0})=5.0 \times 10^{13} \mathrm{~cm}^{-3}$, and the initial number of trapped atoms in a truncated potential $\tilde{N}=1.16 \times 10^{11}$. The time-evolution of evaporative cooling was calculated for 25 s, i.e., the normalized time $\tau$ changes from 0 to 1 .
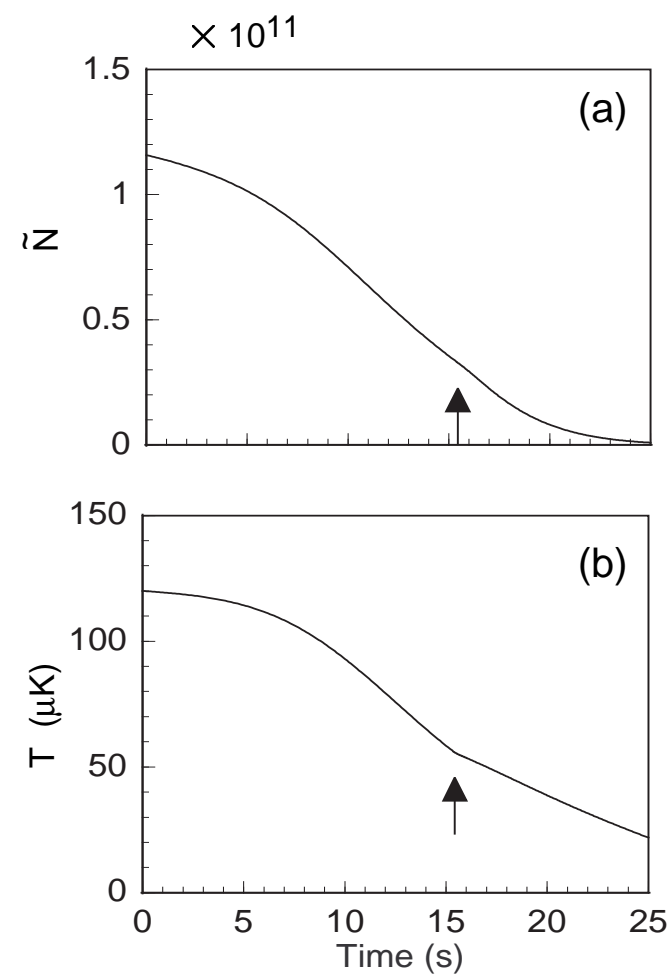

FIG. 1. Time evolution of (a) the total number of trapped atoms $\tilde{N}$, and (b) temperature $T$. The arrow indicates the point at which the BEC transition occurs. The heating due to the dipolar spin relaxation occurs after the BEC transition.

In Figs. 1(a) and 1(b), we show the time evolution of the total number of trapped atoms $\tilde{N}$ and that of temperature $T$, respectively. $\tilde{N}$ decreases monotonically from $1.16 \times 10^{11}$ to $8.7 \times 10^{8}$ during a 25 -s evaporative cooling. The BEC transition occurs at $15.4 \mathrm{~s}(\tau=0.62)$ as indicated by the arrow in the figure. The decrease of temperature (from 120 to $22 \mu \mathrm{K}$ ) is not so large in comparison with that has been demonstrated in alkali-metal atoms [2, 4, 6]. We can see that the cooling speed strongly slows down after the BEC transition in Fig. 1(b), indicating considerable heating occurs. Here we show the calculated results at the BEC transition point: temperature $T=56 \mu \mathrm{K}$, peak density $\tilde{n}(\mathbf{0})=2.0 \times 10^{14} \mathrm{~cm}^{-3}$, number of trapped atoms $\tilde{N}=3.3 \times 10^{10}$, and truncation energy $\epsilon_{t} / k_{\mathrm{B}}=302 \mu \mathrm{K}$. These parameters are quite consistent with the experiment [5], which confirms the validity of our numerical calculations.

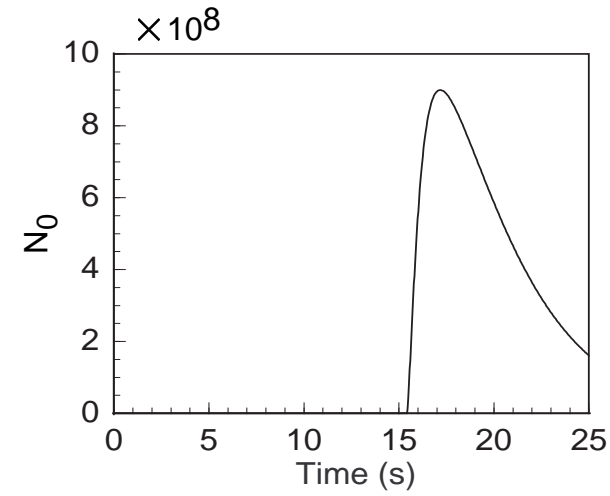

FIG. 2. Time evolution of (a) the total number of trapped atoms $\tilde{N}$, and (b) temperature $T$. The arrow indicates the point at which the BEC transition occurs. The heating due to the dipolar spin relaxation occurs after the BEC transition.

The condensate formation process shows the characteristic feature depicted in Fig. 2. Condensates first grow very rapidly after the transition. We can explain this by the fact that bosonic stimulation in evaporative cooling process 15 strongly accelerates the growing speed, since the density of condensates becomes very high in atomic hydrogen. The number of condensates $N_{0}$ reaches the maximum value at $17.2 \mathrm{~s}(\tau=0.69)$, and then decreases gradually due to dipolar decay. The calculated parameters at this maximum point are: temperature $T=49 \mu \mathrm{K}$, peak density of condensates $n_{p}=4.3 \times 10^{15} \mathrm{~cm}^{-3}$, peak density of noncondensed atoms $\tilde{n}_{n}=1.7 \times 10^{14} \mathrm{~cm}^{-3}$, total number of condensates $N_{0}=9.0 \times 10^{8}$, total number of noncondensed atoms $\tilde{N}_{n}=2.0 \times 10^{10}$, and truncation energy $\epsilon_{t} / k_{\mathrm{B}}=239 \mu \mathrm{K}$. The corresponding condensate fraction at the maximum point of $N_{0}$ is calculated as $f=N_{0} /\left(N_{0}+\tilde{N}_{n}\right)$ to be a small value of $4.3 \%$. These results quantitatively agree with the recent observation of BEC with atomic hydrogen [5].

The number of trapped atoms has been evaluated experimentally by measuring the density-dependent frequency shift of the two-photon $1 S-2 S$ transition in atomic hydrogen [19]. The condensate number, $N_{0} \simeq 10^{9}$ in Ref. [5], was obtained on the assumption that the frequency shift for condensates is as large as that for noncondensed atoms with the same density. If we expect the disappearance of exchange effects in the excitation of condensates as discussed in Ref. [5], the frequency shift for condensates becomes half of that for noncondensed atoms, and many more condensates, $N_{0} \simeq 6 \times 10^{9}$, with an unreasonably higher condensate fraction, $f=25 \%$, are given. The calculated results in Fig. 2 support the evaluation of condensate number which assumes the equal frequency shift for condensates and noncondensed atoms.

Next, we discuss the influence of dipolar loss on evaporative cooling. From Eq. (2), the dipolar relaxation process preferably removes atoms in the region with higher density. Since the removed atoms have lower internal 

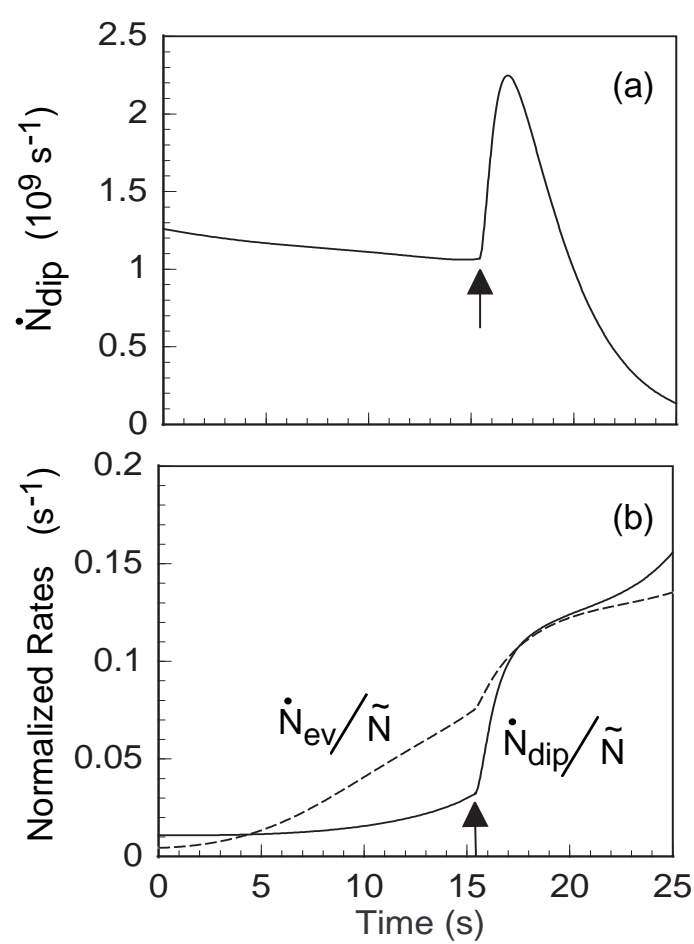

FIG. 3. Time evolution of (a) the dipolar loss rate $\dot{N}_{\text {dip }}$, and (b) normalized rates, $\dot{N}_{\text {ev }} / \tilde{N}$ (dashed line) and $\dot{N}_{\text {dip }} / \tilde{N}$ (solid line). The dipolar loss rate exceeds the evaporation rate at around $17.5 \mathrm{~s}$. The arrow indicates the point at which the BEC transition occurs.

energy in the trapping potential, the dipolar relaxation loss results in system heating. The time evolution of the dipolar loss rate $\dot{N}_{\text {dip }}$ is shown in Fig. 3(a). The curve exhibits the jump structure around the BEC transition point indicated by the arrow. The serious heating after the transition in Fig 1(b) is caused by such drastic increase of dipolar loss known as "relaxation explosion" in hydrogen system [20]. To clearly demonstrate the competition between evaporation and dipolar relaxation processes during evaporative cooling, the normalized rates $\dot{N}_{\text {ev }} / \tilde{N}$ and $\dot{N}_{\text {dip }} / \tilde{N}$ are plotted together in Fig. 3 (b). The evaporation rate is larger than the dipolar loss rate until just after the BEC transition, which indicates that evaporative cooling is sufficiently efficient in this region. The explosively enhanced dipolar-loss rate after the transition finally exceeds the evaporation rate at around $17.5 \mathrm{~s}$, and the resultant heating becomes serious. We note here that this time almost corresponds to the point where the number of condensates shows the maximum value in Fig. 2. The heating-cooling balance therefore limits the producible number of condensates and the corresponding condensate fraction. One can expect that the trapping potential with weaker confinement and the optimized evaporative cooling will move this balance in the direction of a larger condensate production 23.

In conclusion, we have investigated the rf-induced evaporative cooling of magnetically trapped atomic hydrogen on the basis of the kinetic theory of a Bose gas. This approach can be applied over the whole temperature region of evaporative cooling while the earlier investigations based on the classical kinetic theory [7 9, 18,20] fail around the critical point of BEC transition. The calculated results quantitatively agree with the recent BEC experiment of atomic hydrogen. This proves our calculations very useful, and the important future work would be to optimize the cooling trajectory for a larger production of condensates [9]. Our theory, on the other hand, assumes that the system always stays in the quasi-thermal equilibrium states during evaporative cooling. A precise study of the deviation from the quasi-thermal equilibrium [25] would give the limit of the approximation in the theory.

We thank L. Willmann of MIT for his valuable suggestions and discussions.

[1] See, for example, Laser Manipulation of Atoms and Ions, Proceedings of the International School of Physics "Enrico Fermi," Course CXVIII, Varenna, 1991, edited by E. Arimondo, W. D. Phillips, and F. Strumia (North Holland, Amsterdam, 1992); Bose Einstein Condensation, edited by A. Griffin, D. W. Snoke, and S. Stringari (Cambridge University, Cambridge, England, 1995).

[2] M. H. Anderson et al., Science 269, 198 (1995); J. R. Ensher et al., Phys. Rev. Lett. 77, 4984 (1996).

[3] C. C. Bradley et al., Phys. Rev. Lett. 75, 1687 (1995); 78, 985 (1997).

[4] K. B. Davis et al., Phys. Rev. Lett. 75, 3969 (1995); M. -O. Mewes et al., ibid. 77, 416 (1996).

[5] D. G. Fried et al., Phys. Rev. Lett. 81, 3811 (1998).

[6] W. Ketterle and N. J. van Druten, in Advances in Atomic, Molecular, and Optical Physics, edited by B. Bederson and H. Walther (Academic, New York, 1996), Vol. 37, p. 181.

[7] O. J. Luiten, M. W. Reynolds, and J. T. M. Walraven, Phys. Rev. A 53, 381 (1996).

[8] Kirstine Berg-Sørensen, Phys. Rev. A 55, 1281 (1997).

[9] C. A. Sackett, C. C. Bradley, and R. G. Hulet, Phys. Rev. A 55, 3797 (1997).

[10] C. W. Gardiner et al., Phys. Rev. Lett. 79, 1793 (1997); Phys. Rev. A 58, 536 (1998); Phys. Rev. Lett. 81, 5266 (1998).

[11] M. Holland, J. Williams, and J. Cooper, Phys. Rev. A $\mathbf{5 5}, 3670$ (1997).

[12] H. Wu, E. Arimondo, and C. J. Foot, Phys. Rev. A 56, 560 (1997).

[13] D. Jaksch, C. W. Gardiner, and P. Zoller, Phys. Rev. A 56, 575 (1997).

[14] P. W. H. Pinkse et al., Phys. Rev. A 57, 4747 (1998).

[15] M. Yamashita, M. Koashi, and N. Imoto, Phys. Rev. A 59, 2243 (1999). 
[16] R. Walser et al., Phys. Rev. A 59, 3878 (1999).

[17] P. D. Drummond and J. F. Corney, Phys. Rev. A 60, R2661 (1999).

[18] E. L. Surkov, J. T. M. Walraven, and G. V. Shlyapnikov, Phys. Rev. A 49, 4778 (1994); ibid. 53, 3403 (1996).

[19] T. C. Killian et al., Phys. Rev. Lett. 81, 3807 (1998).

[20] T. W. Hijmans et al., Phys. Rev. B 48, 12886 (1993).

[21] Yu. Kagan, B. V. Svitsunov, and G. V. Shlyapnikov, Pis'ma Zh. EkspTeor. Fiz. 42, 169 (1985) [JETP Lett. 42, 209 (1985)].

[22] H. T. C. Stoof et al., Phys. Rev. A 39, 3157 (1989).

[23] L. Willmann, private communication.

[24] M. J. Jamieson, A. Dalgarno, and M. Kimura, Phys. Rev. A 51, 2626 (1995).

[25] R. Côté and V. Kharchenko, Phys. Rev. Lett. 83, 2100 (1999). 